\title{
High-pt Jet Studies at CMS
}

\author{
Joanna WENG $* \dagger$ \\ Institute for Particle Physics (IPP), ETH Zurich \\ E-mail: joanna.weng@cern.ch
}

\begin{abstract}
We present an update to CMS plans to search for physics beyond the standard model using dijets. We study jet cleanup, jet response versus $\eta$, optimization of $|\eta|$ cuts, and the dijet mass resolution. Estimates are presented for both the QCD background and signals of new physics with a focus on the integrated luminosities $10 \mathrm{pb}^{-1}, 100 \mathrm{pb}^{-1}$, and $1 \mathrm{fb}^{-1}$ expected early in LHC running. The inclusive cross section as a function of jet $p_{T}$ is a first simple measure of QCD dijets which is sensitive to a $3 \mathrm{TeV}$ contact interaction with only $10 \mathrm{pb}^{-1}$. With the dijet mass distribution we expect to be able to observe dijet resonances with large cross sections, such as a $2 \mathrm{TeV}$ excited quark which produces a convincing signal with only $100 \mathrm{pb}^{-1}$. With the dijet ratio, a simple angular measurement, we expect to be able to discover a contact interaction scale $\Lambda^{+}$of 4,7 and $10 \mathrm{TeV}$ for integrated luminosities of $10 \mathrm{pb}^{-1}, 100 \mathrm{pb}^{-1}$, and $1 \mathrm{fb}^{-1}$ respectively. Using the dijet ratio we can discover or confirm a dijet resonance, and eventually measure its spin. With $100 \mathrm{pb}^{-1}$ a $2 \mathrm{TeV}$ resonance with the production rate of an excited quark produces a convincing signal in the dijet ratio.
\end{abstract}

Physics at LHC 2008

29 September - October 4, 2008

Split, Croatia

*Speaker.

$\dagger$ On behalf of the CMS Collaboration 
This analysis uses a CMS cone algorithm with a cone size $\mathrm{R}=0.5$ to reconstruct jets. Three kinds of jets are discussed: GenJets are from the Monte Carlo particles, CaloJets are observed at the calorimeter level after a full CMS detector simulation, and corrected CaloJets have Lorentz vectors that have been scaled to equal the GenJet on average. Dijets are the two jets with largest transverse momentum, $p_{T}$, in the event. All jets used in this analysis satisfy the requirement $|\eta|<1.3$. Further studies within this analysis demonstrate that jet response in this $\eta$ region is expected to vary smoothly and can be measured reliably in data via dijet $p_{T}$ balance $[1,4]$.

\section{Inclusive Jet $\mathbf{p}_{T}$ from QCD and Contact Interactions}

Inclusive jet $p_{T}$ is a QCD measurement that is sensitive to new physics. For the cross section we count all jets inside a $p_{T}$ bin and $\eta$ interval and divide by bin width and luminosity. Fig. 1 shows that contact interactions [5] create a large rate at high $p_{T}$, and discovery is possible with only $10 \mathrm{pb}^{-1}$. Errors are expected to be dominated by jet energy scale uncertainty (10\%) in early running $\left(10 \mathrm{pb}^{-1}\right)$. Fig. 1 shows that a contact scale $\Lambda^{+}=3 \mathrm{TeV}$ would be observable despite jet energy errors for $p_{T}>1 \mathrm{TeV}$. Statistics and PDFs give smaller uncertainties. For $10 \mathrm{pb}^{-1}$ we can discover a contact interaction beyond the Tevatron exclusion of $\Lambda^{+}<2.7 \mathrm{TeV}$ [2].
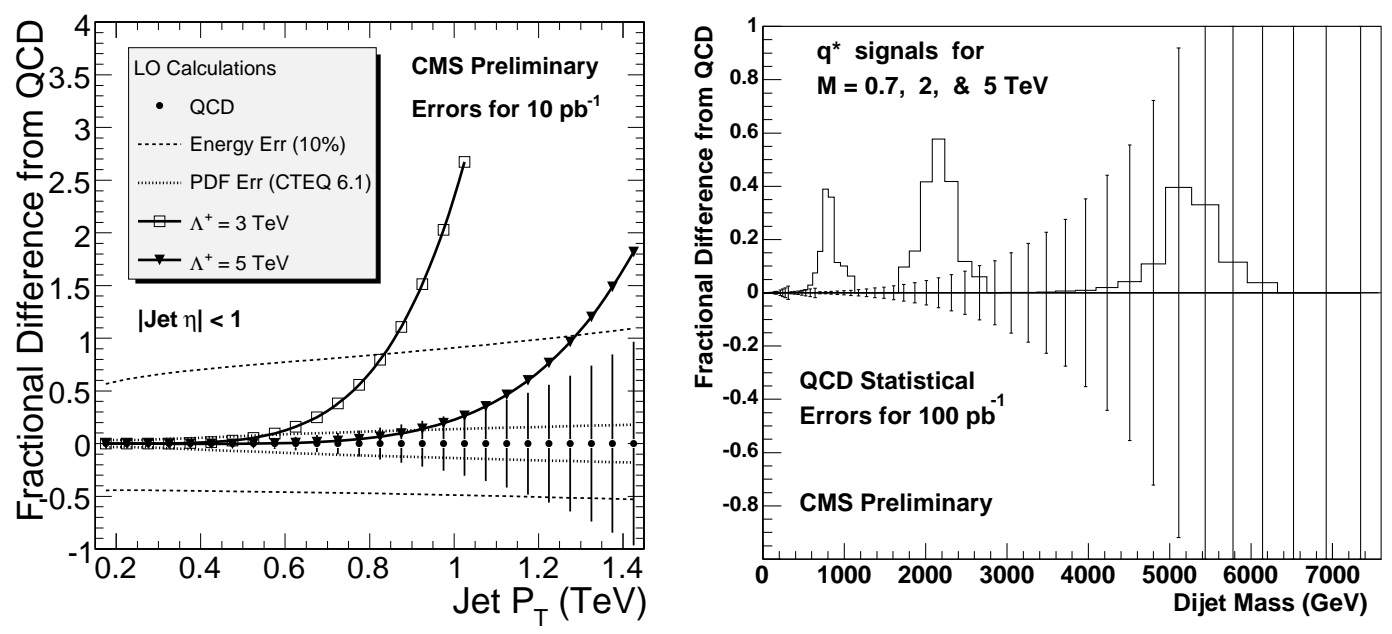

Figure 1: Left) Contact interactions and systematic uncertainties in jet cross section for $10 \mathrm{pb}^{-1}$. Right) Dijet resonances compared to QCD.

\section{Dijet Mass from QCD and Resonances}

The dijet mass distribution will be used to search for dijet resonances. We can use a fit or a prediction for the QCD background. Fig. 1 shows a simulation of QCD and of dijet resonances modeled using a $Z^{\prime}$ shape. Strongly produced resonances have large enough rate to be seen above the background. Fig. 1 shows we expect a convincing signal for a $2 \mathrm{TeV}$ excited quark ( $\mathrm{q}^{*}$ ) with $100 \mathrm{pb}^{-1}$, well beyond the Tevatron exclusion of $M<0.78 \mathrm{TeV}$ in the dijet channel $[3,7]$. 


\section{Dijet Ratio from QCD, Contact Interactions and Resonances}

The dijet ratio uses dijet angular distributions to search for new physics. We have optimized the dijet ratio for a contact interaction search in the CMS barrel with $100 \mathrm{pb}^{-1}$. The old dijet ratio used by the D0 search [2] and prior CMS analysis [6] was $N(|\eta|<0.5) / N(0.5<|\eta|<1.0)$, and the new dijet ratio is $N(|\eta|<0.7) / N(0.7<|\eta|<1.3)$, where $\mathrm{N}$ is the number of dijets for which each of the two jets satisfies the $|\eta|$ requirement in parenthesis. Detailed studies show that the dijet ratio for QCD agrees for GenJets and corrected CaloJets. QCD is flat at 0.6 for the old ratio and flat at 0.5 for the new ratio up to a dijet mass of about $6 \mathrm{TeV}$, as can be seen in Fig. 2
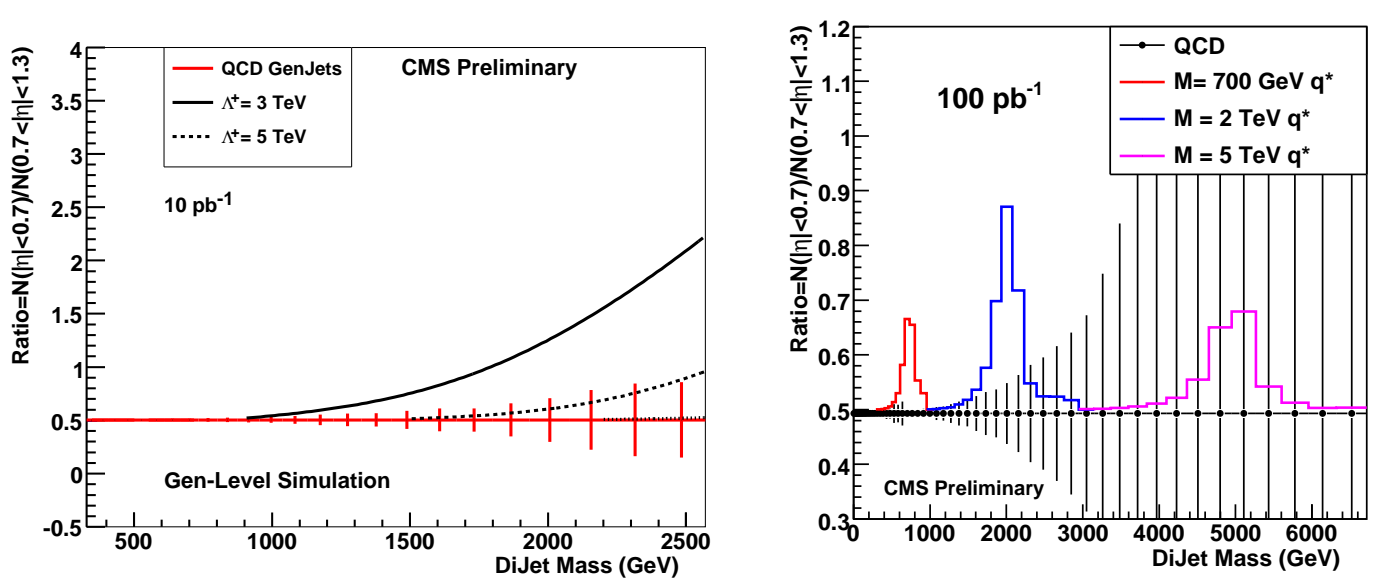

Figure 2: Left) Dijet ratio from QCD and contact interactions for $10 \mathrm{pb}^{-1}$. Right) Dijet ratio for excited quarks compared to QCD $\left(100 \mathrm{pb}^{-1}\right)$.

Our sensitivity to a contact interaction with the dijet ratio is summarized in table 1 using the same methodology as previous CMS estimates [6]. The new definition of the dijet ratio increases our discovery sensitivity to $\Lambda^{+}$by 1-2 TeV depending on luminosity.

\begin{tabular}{|c|c|c|c|c|c|c|}
\hline & \multicolumn{3}{|c|}{ Excluded $\Lambda(\mathrm{TeV})$} & \multicolumn{3}{c|}{ Discovered $\Lambda(\mathrm{TeV})$} \\
\cline { 2 - 7 } & $10 \mathrm{pb}^{-1}$ & $100 \mathrm{pb}^{-1}$ & $1 \mathrm{fb}^{-1}$ & $10 \mathrm{pb}^{-1}$ & $100 \mathrm{pb}^{-1}$ & $1 \mathrm{fb}^{-1}$ \\
\hline DO and PTDR $\eta$ cuts & $<3.8$ & $<6.8$ & $<12.2$ & $<2.8$ & $<4.9$ & $<9.1$ \\
\hline Optimized $\eta$ cuts & $<5.3$ & $<8.3$ & $<12.5$ & $<4.1$ & $<6.8$ & $<9.9$ \\
\hline
\end{tabular}

Table 1: Sensitivity to contact interactions with $10 \mathrm{pb}^{-1}, 100 \mathrm{pb}^{-1}$, and $1 \mathrm{fb}^{-1}$ for both the old $\eta$ cuts and the optimized $\eta$ cuts. Estimates include statistical uncertainties only.

In Fig. 2 we show our sensitivity using the dijet ratio to a resonance signal in the presence of the QCD background. The resonances are all normalized with the cross section of an excited quark for $|\eta|<1.3$. There is a convincing signal for a $2 \mathrm{TeV}$ resonance with $100 \mathrm{pb}^{-1}$. We expect that with sufficient luminosity the dijet ratio can be used to measure the resonance spin. More details on this study can be found in [1]. 


\section{References}

[1] CMS Collaboration, CMS PAS SBM-07-001 (2007).

[2] Abbott, B. and others,D0 Collaboration, Phys. Rev. Lett. 82 (1999). [arXiv:hep-ex/9807014]

[3] Abe, F. and others,CDF Collaboration, Phys. Rev. D55 (1997). [arXiv:hep-ex/9702004]

[4] CMS Collaboration, CMS PAS JME-07-002 (2007).

[5] Eichten, E. and Lane, K. D. and Peskin, M. E., Phys. Rev. Lett. D50 (1983).

[6] Esen, S. and Harris, R., CMS Note 2006/071 (2006).

[7] Abazov, V. M. and others, D0 Collaboration, Phys. Rev. D69 (2004). [arXiv:hep-ex/0308033] 